\title{
Understanding the Impact of Line-of-Sight in the Ergodic Spectral Efficiency of Cellular Networks
}

\author{
Celia García-Corrales, Francisco J. Martin-Vega, F. Javier Lopez-Martinez, \\ Francisco J. Cañete and José F. Paris \\ Dpto. de Ingeniería de Comunicaciones \\ Universidad de Málaga. Andalucía Tech \\ 29071 Málaga. Spain \\ \{celia, fjmvega, fjlopezm, francis, paris\}@ic.uma.es
}

\begin{abstract}
In this paper we investigate the impact of lineof-sight $(\mathrm{LOS})$ condition in the ergodic spectral efficiency of cellular networks. To achieve this goal, we have considered the $\kappa-\mu$ shadowed model, which is a general model that provides an excellent fit to a wide set of propagation conditions. To overcome the mathematical complexity of the analysis, we have split the analysis between large and small-scale effects. Building on the proposed framework, we study a number of scenarios that range from heavily-fluctuating LoS to deterministic-LoS. Finally, we shed light on the interplay between fading severity and spectral efficiency by means of the amount of fading.

Index Terms-Wireless communications, average spectral efficiency, $\kappa-\mu$ shadowed fading model, amount of fading
\end{abstract}

\section{INTRODUCTION}

A promising approach to cope with the ever-increasing capacity demand in cellular networks is to increase the base station (BS) density, which reduces the link distances, and thus improve overall system performance [1]. Therefore, the possibility of having a line-of-sight (LoS) transmission is higher, and not only in typical rural areas, but also in urban scenarios.

The distribution of the Signal to Interference plus Noise Ratio (SINR) on a wireless link is an important basis for studying the capacity in a cellular network. Nevertheless, the uncertainty of the locations of both BSs and users, and the high number of interfering BSs complicate the problem at hand. The mathematical analysis using Poisson Point Process (PPP) has proved to be a powerful and accurate tool for the research in these fields [2, 3]. This approach captures the spatial randomness of wireless networks and allows to include other sources of uncertainties such as fading and shadowing.

In these environments, reflections, diffraction and scattering lead to a complex propagation scenario for the signal travelling from a transmitter to a receiver. To deal with it, a widely used approximation is to classify the propagation mechanisms in large-scale phenomena (characteristics over hundreds of wavelengths) and small-scale phenomena (on the order of a wavelength). This separation relies on the fact that, for a representative amount of time, the large-scale phenomena remain essentially unchanged and only small-scale variations arise. This fact was exploited in [4] to develop a framework that splits the analysis in two parts: i) the large-scale analysis, based on the concept of local average SINR; and ii) the small scale analysis, which focused on Rayleigh fading.

Small-scale analysis relies on statistical models that attempt to reproduce the variations of the channel effects. The $\kappa$ - $\mu$ shadowed model, introduced in [5], has proven to be a powerful and general model that covers propagation conditions ranging from very favorable to worse-than-Rayleigh fading. The model considers the received signal as formed by one or several groups of multipath signals, each being able to have a main component whose amplitude can fluctuate. It includes Rayleigh, Rice, Rice shadowed, Nakagami- $m, \kappa-\mu$ and $\eta-\mu$ as particular cases $[6,7]$. Changing its fading parameters $(\kappa$, $\mu$ and $m$ ), the model can study non-line-of-sight scenarios (NLoS) and line-of-sight scenarios (LoS) with different characteristics.

The potential of generalized fading models to obtain insights about wireless networks in realistic propagation conditions has been proven with a number of recent works. The analysis of cellular systems with $\kappa-\mu$ shadowed fading has been firstly analyzed with the aid of stochastic geometry in [8]. In [9], the outage probability and spectral efficiency is extended for the case of Device-to-Device (D2D) communications under cellular and D2D modes. The case of $5 \mathrm{G}$ heterogeneous cellular networks is investigated in [10].

In this work, we follow a different approach than those in [8-10], which was presented in [4] for Rayleigh fading (i.e., for NLoS set-ups). We extend their work by assuming a wider set of propagation conditions using the $\kappa$ - $\mu$ shadowed fading model. Several LoS scenarios, with deterministic and fluctuating main components and different associated powers, are studied and compared with NLoS environments.

More specifically, in this paper we provide the following contributions:

1) We extend the approach in [4] to consider $\kappa$ - $\mu$ shadowed fading and investigate the effect of LoS in the spectral efficiency, through several scenarios that range from heavily fluctuating LoS to large deterministic LoS.

2) We derive a closed-form expression for the amount of fading (AoF) of the $\kappa-\mu$ shadowed fading model.

3) We analyze the interplay between fading severity and spectral efficiency by means of the AoF. 
4) We study the effect of the main parameters of the fading distribution on the spectral efficiency and AoF.

The remainder of this paper is structured as follows. Section II introduces the network model and the $\kappa$ - $\mu$ shadowed fading model, together with the selection of scenarios to study. In Section III, we present the spectral efficiency expressions used and the results for LoS and NLoS scenarios. We also consider the AoF as an easy way to foresee the capacity behavior associated to a certain set of propagation conditions. The impact of the fading parameters of $\kappa-\mu$ shadowed channel model is analyzed in Section IV. Finally, conclusions are drawn in Section $\mathrm{V}$.

\section{SySTEM MODEL}

We here assume a system model for the downlink of a cellular system similar to the one recently introduced in [4]. Thus, we consider a cellular network set-up with omnidirectional antennas, where the signals are subject to path loss with exponent $\eta>2$ and shadowing, and all BSs experience the same $\eta$. The BSs are spatially distributed following a PPP model, so the shadowing is implicitly captured by the Poisson nature of the network. The analysis focuses on the typical user, which is a randomly selected user. Thanks to Slivnyak's theorem, this user can be considered to be placed at the origin without loss of generality [2]. Let us denote by $r_{k}$ the distance between such an user and the kth BS, being $r_{0}<\ldots<r_{k}<r_{k+1}<\ldots$. So, the serving BS for the user of interest is the one located at $r_{0}$.

We will assume that the user and the BSs have a single antenna, so the communication is SISO. The signal received by the user at symbol $\mathrm{n}$ is [4]:

$$
y[n]=\sqrt{P r_{0}^{-\eta}} H_{0}[n] s_{0}[n]+z[n]
$$

with

$$
z[n]=\sum_{k=1}^{\infty} \sqrt{\operatorname{Pr}_{k}^{-\eta}} H_{k}[n] s_{k}[n]+v[n]
$$

where $P$ is the power measured at $1 \mathrm{~m}$ from the BS transmitter; $s_{k} \sim N_{c}(0,1)$ is the signal transmitted by the $k_{t h} \mathrm{BS}, H_{k}$ is the associated fading coefficient $\left(\left\{H_{k}\right\}_{k=0}^{\infty}\right.$ are independent and of unit power) and $z$ is the aggregate interference from the rest of BSs plus thermal noise, $\nu \sim N_{c}\left(0, N_{0}\right)$. As justified in [4], $z$ can be accurately approximated as Gaussian, giving up the small-scale variations in its power.

This makes possible to define the instantaneous SINR over a symbol, conditioned on $\left\{r_{k}\right\}_{k=0}^{\infty}$, as [4]:

$$
S I N R=\frac{P r_{0}^{-\eta}\left|H_{0}\right|^{2}}{P \sum_{k=1}^{\infty} r_{k}^{-\eta}+N_{0}}=\rho\left|H_{0}\right|^{2}
$$

where $\rho$, related to the large scale, can be seen as the localaverage SINR for the typical user (over the small-scale fading only, hence it is valid for a region small enough to consider that the large-scale behavior, pathloss and the conventional notion of shadowing, do not change significantly). Therefore, the separation between large-scale propagation phenomena and small-scale multipath fading largely relaxes the complexity of the system characterization.

With all the previous considerations, an approximation to the ergodic spectral efficiency can be written as [4]:

$$
C=\mathrm{E}\left[\log _{2}\left(1+\frac{P r_{0}^{-\eta}\left|H_{0}\right|^{2}}{P \sum_{k=1}^{\infty} r_{k}^{-\eta}+N_{0}}\right)\right]
$$

with $C \leq C_{\text {exact }}$, being $C_{\text {exact }}$ the achievable spectral efficiency if the BS would know the fading states of all the interfering links $[4,11]$. Since this situation is not realistic in practical systems, we will focus on $C$ according to (4) in this work. Henceforth, this approximation will be used whenever talking about spectral efficiency.

As opposed to [4], where Rayleigh fading was assumed, we here aim to consider more advanced fading models that allow for including the effect of LoS propagation between the BS and the user. Thus, for the small-scale fading we assume the popular and versatile $\kappa-\mu$ shadowed fading model $^{1}[5,12]$ with parameters:

- $\mu$ : the number of clusters.

- $\kappa$ : the ratio between the power of the dominant (LoS) components and the power of the scattered waves.

- $m$ : the fluctuation degree of the dominant (LoS) components.

The $\kappa$ - $\mu$ shadowed fading model is well-suited for propagation conditions in a wide spectrum, and includes as special cases simpler and widespread models as Rayleigh, Nakagami, Rician, or Rician Shadowed just to name a few. In order to analyze the impact in the capacity when there is a LoS component, we will define several propagation scenarios: NLoS, conventional (deterministic) LoS, and fluctuating LoS, which correspond to values of the fading parameters presented in Table I. In all cases, for the sake of simplicity, we suppose a single cluster (i.e. $\mu=1$ ). Later, in section IV, the impact of each of the three fading parameters will be further investigated.

\section{Spatial Average of the Spectral EfFiciency}

The ergodic spectral efficiency of a user with $\bar{\gamma}=\rho$ as its local-average SINR can be computed using [12]:

$$
C(\rho)=\log _{2}(e) \sum_{i=0}^{M} C_{i} e^{1 /\left(\rho \hat{\Omega}_{i}\right)} \sum_{k=0}^{m_{i}-1} \frac{\Gamma\left(-k, 1 /\left(\rho \hat{\Omega}_{i}\right)\right)}{\left(\rho \hat{\Omega}_{i}\right)^{k}}
$$

where $\Omega_{i}=\bar{\gamma} \widehat{\Omega}_{i}=\rho \widehat{\Omega}_{i}, C_{i}, M$ and $m_{i}$ are given in [Table I, [12]].

The achievable spectral efficiency for a user at the origin versus the distance to the serving BS is presented in Fig. 1. It shows the scenarios in Table I for an interference-limited

\footnotetext{
${ }^{1}$ We must clarify that the term shadowing in the $\kappa-\mu$ shadowed fading distribution is not linked to the conventional notion of large-scale shadowing. Instead, this model captures any sort of amplitude fluctuation in the specular waves that may occur in a smaller-scale. Thus, the use of such model is coherent with the ergodic setting here analyzed.
} 
Table I: Propagation scenarios to study

\begin{tabular}{|l|c|c|c|}
\hline Scenario & $\mu$ & $\kappa$ & $m$ \\
\hline $\begin{array}{l}\text { NLoS } \\
\text { (Rayleigh) }\end{array}$ & 1 & 0 & $\infty(20)$ \\
\hline $\begin{array}{l}\text { Heavily-Fluctuating LoS: hfLoS } \\
\text { (Rician shadowed) }\end{array}$ & 1 & 10 & 2 \\
\hline $\begin{array}{l}\text { Mildly-Fluctuating LoS: mfLoS } \\
\text { (Rician shadowed) }\end{array}$ & 1 & 10 & 5 \\
\hline $\begin{array}{l}\text { Low Deterministic LoS: ldLoS } \\
\text { (Rician) }\end{array}$ & 1 & 1 & $\infty(20)$ \\
\hline $\begin{array}{l}\text { Medium Deterministic LoS: mdLoS } \\
\text { (Rician) }\end{array}$ & 1 & 3 & $\infty(20)$ \\
\hline $\begin{array}{l}\text { Large Deterministic LoS: dLoS } \\
\text { (Rician) }\end{array}$ & 1 & 10 & $\infty(20)$ \\
\hline
\end{tabular}

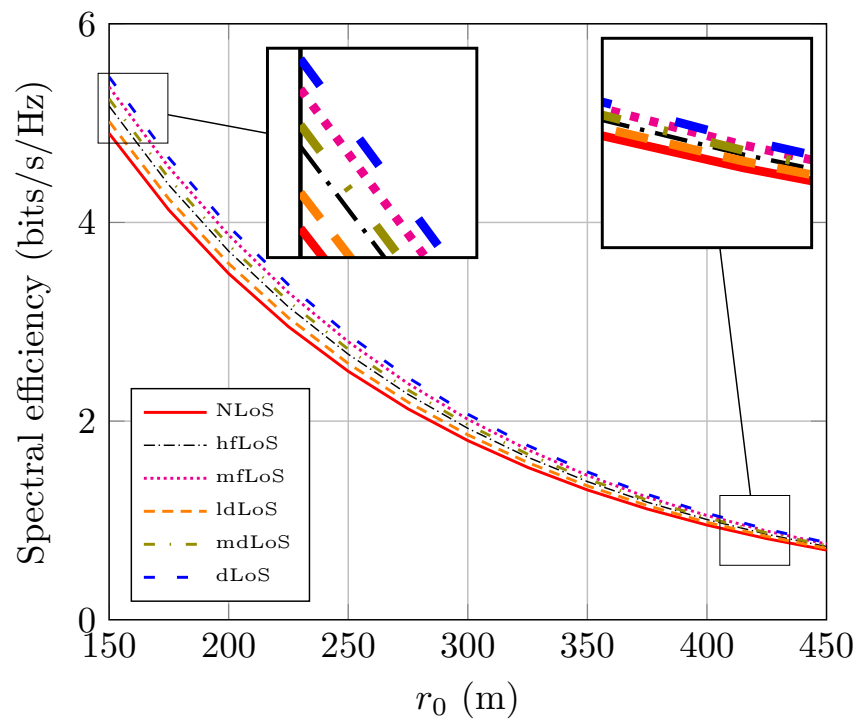

Figure 1: Spectral efficiency vs $r_{0}$ for scenarios in Table I.

network with 100 interfering BSs, $\eta=3.8$ and $\lambda=2 \mathrm{BSs} / \mathrm{km}^{2}$ [4].

As the user moves away from the serving BS, the signal arriving at the receiver is weaker and, therefore, spectral efficiency is decreased. For distances above $400 \mathrm{~m}$, the absolute difference between spectral efficiency among NLoS and LoS scenarios is almost negligible, but the relative difference is maintained. If we measure the impact of LoS condition with respect to the NLoS case as:

$$
\text { impact }(\%)=\frac{C_{\mathrm{xLoS}}-C_{\mathrm{NLoS}}}{C_{\mathrm{NLoS}}} \times 100
$$

where xLOS denotes any of the scenarios listed in Table I, the impact in spectral efficiency for dLoS in Fig. 1 is above $10 \%$ for all the distances, moving from $14.7 \%$ for $r_{0}=300 \mathrm{~m}$ to $10.7 \%$ for $r_{0}=450 \mathrm{~m}$.

The path loss exponent, $\eta$, also plays an important role. The higher $\eta$, the larger attenuation in the environment, so there is less aggregate interference and higher spectral efficiency,

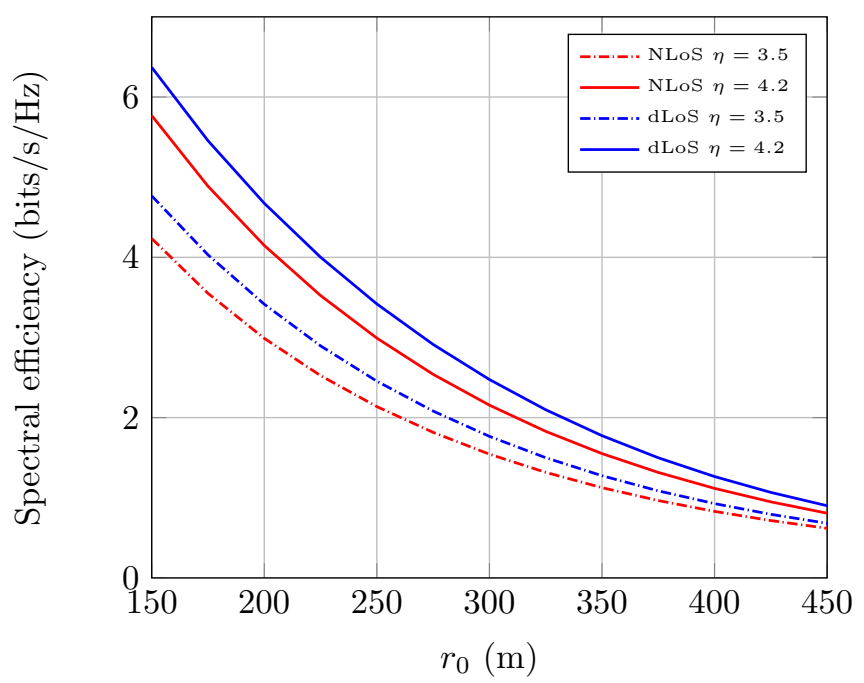

Figure 2: Spectral efficiency vs $r_{0}$ for scenarios NLoS and dLoS in Table I for $\eta=3.5$ and $\eta=4.2$.

as shown in Fig.2. The relative increase in spectral efficiency for $r_{0}=150 \mathrm{~m}$ from $\eta=3.5$ to $\eta=4.2$ is $29.1 \%$ for NLoS and $32 \%$ for dLoS. The impact of dLoS is between $10 \%$ and $15 \%$ for all the distances from the serving BS and the set of $\eta$ studied.

The above values are computed conditioned to a specific network spatial realization. However, it is of interest to provide a single quantity that represents the spectral efficiency of the whole network. This corresponds to the average of all per-user spectral efficiencies in any realization of the network, named as the spatially average ergodic spectral efficiency [4]:

$$
\bar{C}=\int_{0}^{\infty} C(\theta) d F_{\rho}(\theta)
$$

For interference-limited Poisson networks, the cumulative distribution function $F_{\rho}()$ can be approximated by [eq. 22, [4]]:

$$
\left\{\begin{array}{llrl}
F_{\rho}(\theta) & \simeq e^{s^{*} / \theta} & & 0 \leq \theta \leq \frac{s^{*}}{\log (1-\sin \delta)} \\
F_{\rho}(\theta) & \approx 1-\operatorname{sinc} \delta & & \frac{s^{*} \log (1-\sin \delta)}{\ln \delta} \leq \theta \leq 1 \\
F_{\rho}(\theta) & =2-\theta^{-\delta} \operatorname{sinc} \delta & & \theta \geq 1
\end{array}\right.
$$

There is also a slightly more accurate approximation, [eq.18, [4]], but it is much more complex for further analytical calculations. In Fig. 3, it is shown the spatially average ergodic spectral efficiency obtained using [eq.18, [4]], [eq.22, [4]] and PPP Monte Carlo simulations, for NLoS and dLoS scenarios. The improvement from NLoS to dLoS is around 11-12\% within the three methods. There are just slight differences in the results, so that it is justified to rely on (7) henceforth to obtain $\bar{C}$ due to its greater simplicity. The values obtained by (7) are slightly pessimistic compared to those using [eq.18, [4]], but insightful enough to understand the impact of LoS in the average ergodic spectral efficiency. Besides, both are lower bounds of the actual average spectral efficiency obtained using 


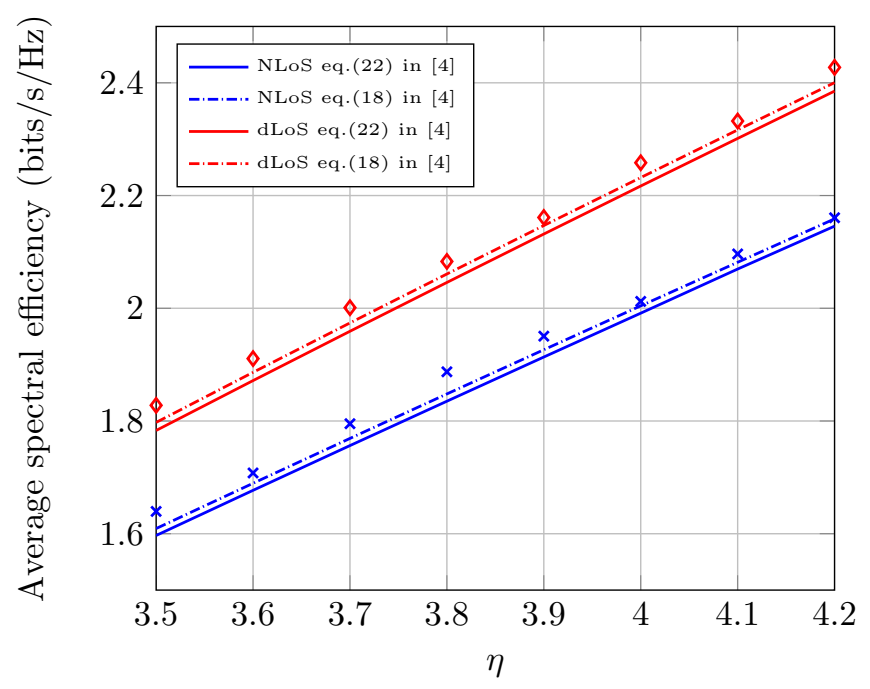

Figure 3: Spatially average ergodic spectral efficiency as a function of $\eta$ for NLoS and dLoS scenarios, with two approximations for $F_{\rho}()$ [eq.(18), eq.(22), [4]]. Markers correspond to Monte Carlo simulations.

\section{Monte Carlo simulations.}

We now evaluate the average spectral efficiency for the set of scenarios previously defined in Table I. LoS scenarios under study can be classified in fluctuating $\operatorname{LoS}$ and deterministic LoS. The former is related to situations where there is a large power in the main components $(\kappa=10)$ but with several fluctuation degrees: heavy $(m=2)$, mild $(m=5)$ and nearly zero or deterministic ${ }^{2}(m=20)$. The latter is related to environments where there is no fluctuation in the main components $(m=20)$ but its power can be low $(\kappa=1)$, medium $(\kappa=3)$ or high $(\kappa=10)$.

Fig. 4 and Fig. 5 show the spatially average spectral efficiency for LoS fluctuating scenarios and deterministic ones respectively. In both cases, all the results are confined within the minimum corresponding to NLoS and the maximum to dLoS. As previously indicated, simulated values obtained through Monte Carlo simulations provide slightly larger results due to the approximation for $C$ used in (4).

After inspecting the results, it is easy to observe which scenario leads to a higher $\bar{C}$. For example, for $\eta=4$, $\bar{C}_{m f L o S}=2.18 \mathrm{bit} / \mathrm{s} / \mathrm{Hz}$ and $\bar{C}_{m d L o S}=2.13 \mathrm{bit} / \mathrm{s} / \mathrm{Hz}$, so $\mathrm{mfLoS}$ is better. However, it would be desirable to establish a relationship between the fading parameters (and ultimately, the fading severity) and the capacity degradation. We aim to illustrate such effect by using the classical metric known as Amount of Fading (AoF) [13].

The AoF is a simple performance measure for describing the severity of fading in wireless systems, $\operatorname{AoF}=\operatorname{Var}[\gamma] /(E[\gamma])^{2}$, defined as the ratio between the variance and the squared mean of the signal power $\gamma$. For statistic channel models, AoF can be evaluated by means of

\footnotetext{
${ }^{2}$ As justified in [12], the LoS fluctuation becomes deterministic as $m \rightarrow$ $\infty$. In practice, it suffices to consider sufficiently large $m$ (i.e. $m=20$ ) for approximating the deterministic LoS case (i.e. Rician fading).
}

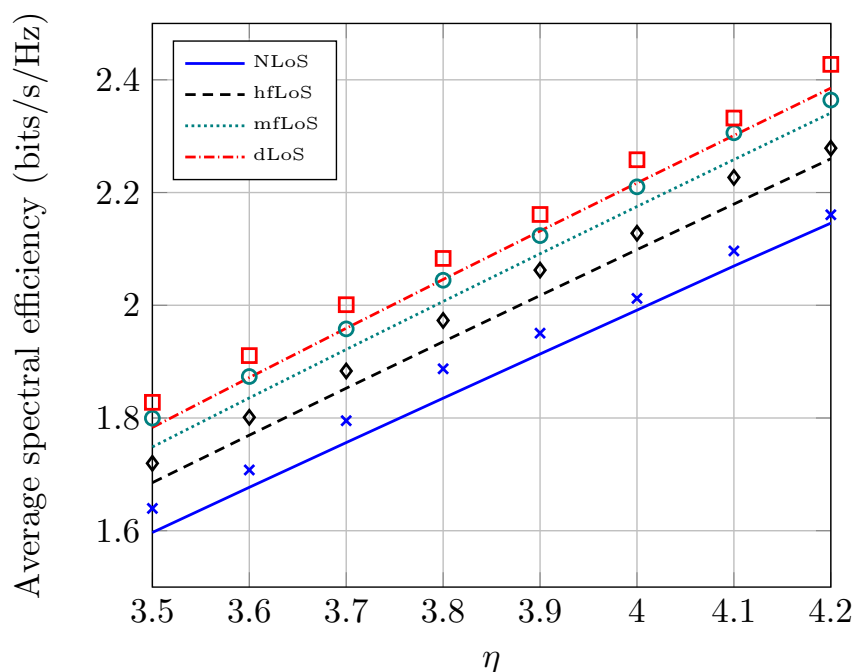

Figure 4: Spatially average ergodic spectral efficiency as a function of $\eta$ for NLoS and fluctuating LoS scenarios. Markers correspond to Monte Carlo simulations.

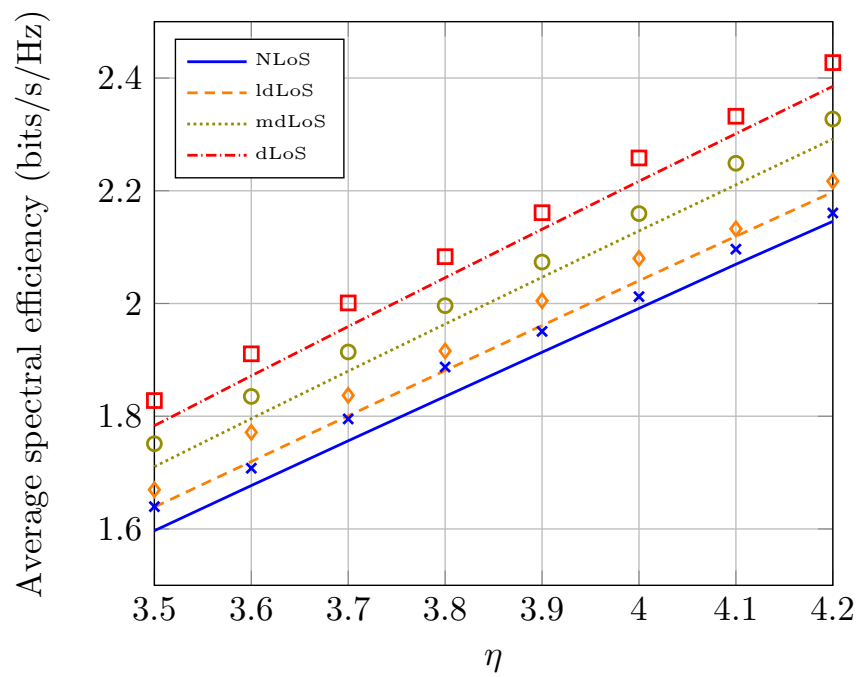

Figure 5: Spatially average ergodic spectral efficiency as a function of $\eta$ for NLoS and deterministic LoS scenarios. Markers correspond to Monte Carlo simulations.

the moment-generating function (MGF) of $\gamma$ as [13]:

$$
A o F=\frac{d^{2} M_{\gamma}(s) /\left.d s^{2}\right|_{s=0}-\left.\left(d M_{\gamma}(s) / d s\right)^{2}\right|_{s=0}}{\left.\left(d M_{\gamma}(s) / d s\right)^{2}\right|_{s=0}}
$$

Following this, for $\kappa-\mu$ shadowed fading model the AoF admits the following closed-form expression:

$$
\begin{aligned}
\text { AoF } & =\frac{1}{\mu} \frac{1+2 \kappa}{(1+\kappa)^{2}}+\frac{1}{m} \frac{\kappa^{2}}{(1+\kappa)^{2}} \\
& =\frac{1}{\mu}\left[1-\frac{\kappa^{2}}{(1+\kappa)^{2}}\right]+\frac{1}{m} \frac{\kappa^{2}}{(1+\kappa)^{2}}
\end{aligned}
$$

This AoF ranges from 0 (no fading) to 2. For the case of integer fading parameters $m$ and $\mu$, the maximum AoF is 1 , 
Table II: $\kappa-\mu$ shadowed parameters, AoF and spatially averaged spectral efficiency

\begin{tabular}{|c|c|c|c|c|c|c|}
\hline & $\mu$ & $\kappa$ & $m$ & $\mathrm{AoF}$ & $\bar{C}_{\eta=3.5}$ & $\bar{C}_{\eta=4.2}$ \\
\hline NLoS & 1 & 0 & $\infty(20)$ & 1 & 1.60 & 2.15 \\
\hline & 1 & 10 & 1 & 1 & 1.60 & 2.15 \\
\hline ldLoS & 1 & 1 & $\infty(20)$ & 0.76 & 1.64 & 2.20 \\
\hline hfLoS & 1 & 10 & 2 & 0.59 & 1.69 & 2.26 \\
\hline & 1 & 3.77 & 5 & 0.50 & 1.70 & 2.28 \\
\hline & 2 & 0 & $\infty(20)$ & 0.50 & 1.71 & 2.30 \\
\hline mdLoS & 1 & 3 & $\infty(20)$ & 0.47 & 1.71 & 2.30 \\
\hline mfLoS & 1 & 10 & 5 & 0.34 & 1.75 & 2.34 \\
\hline dLoS & 1 & 10 & $\infty(20)$ & 0.21 & 1.78 & 2.38 \\
\hline & 5 & 0 & $\infty(20)$ & 0.20 & 1.79 & 2.39 \\
\hline & 3 & 3.10 & 10 & 0.20 & 1.79 & 2.39 \\
\hline
\end{tabular}

coincident with the Rayleigh case. An easy way to foresee which scenario will provide a larger spectral efficiency could be calculating its corresponding AoF. As we can observe in Table II, the lower the AoF, the better the capacity. We can also see that scenarios with equivalent AoF, have a similar spectral efficiency.

\section{IMPACT OF $\kappa-\mu$ Shadowed MOdEL PARAMETERS ON THE AVERAGE SPECTRAL EFFICIENCY}

In this section, the impact of each of the fading parameters $(\mu, \kappa, m)$ on the spatially averaged spectral efficiency will be analyzed. For the sake of brevity, we will consider a fixed value of $\eta=3.8$, as the behavior for other values of $\eta$ is formally equivalent.

\section{A. Impact of $\mu$}

All the scenarios in Table I are represented by a single cluster. Here, the influence of increasing the number of clusters $\mu$ is studied. Note that the parameter $\mu$ can also be regarded as the number of diversity branches in a multiantenna receiver based on maximal ratio combining (MRC). The remainder parameters are set to: LoS scenarios $(\kappa=1,3,10)$ with high fluctuation $(m=2)$ and no fluctuation $(m=20)$, and NLoS situation $(\kappa=0, m=20)$. For all of them, $\mu$ has a positive impact on $\bar{C}$, especially from 1 to 5 , as shown in Fig. 6 . Deterministic LoS and NLoS environments tend to a similar maximum $\bar{C}$ as $\mu$ increases, whereas, with high fluctuation, it depends on $\kappa$ value. This also can be interpreted looking at the AoF expression in (9), where the first term loses importance as $\mu$ increases and the second one, which depends inversely on $m$, grows with $\kappa$. Fluctuating LoS scenarios get lower $\bar{C}$ than NLoS for $\mu>2$.

\section{B. Impact of $m$}

This parameter only affects to LoS scenarios, as it represents a potential fluctuation in the dominant specular (or LoS) components, due to rapid human-body shadowing. Such fluctuation

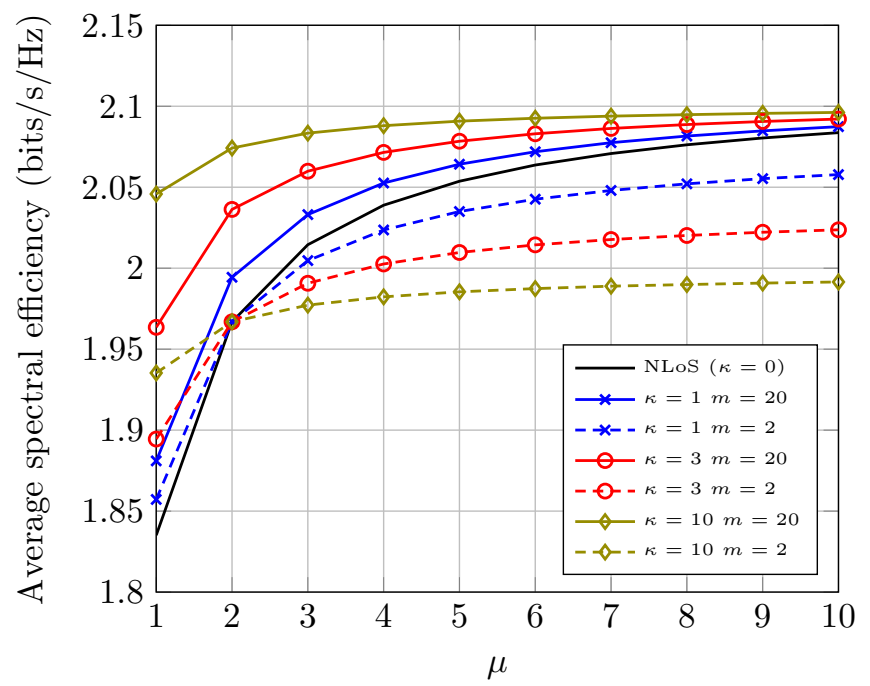

Figure 6: Impact of $\mu$ on the spatially averaged spectral efficiency $(\eta=3.8)$.

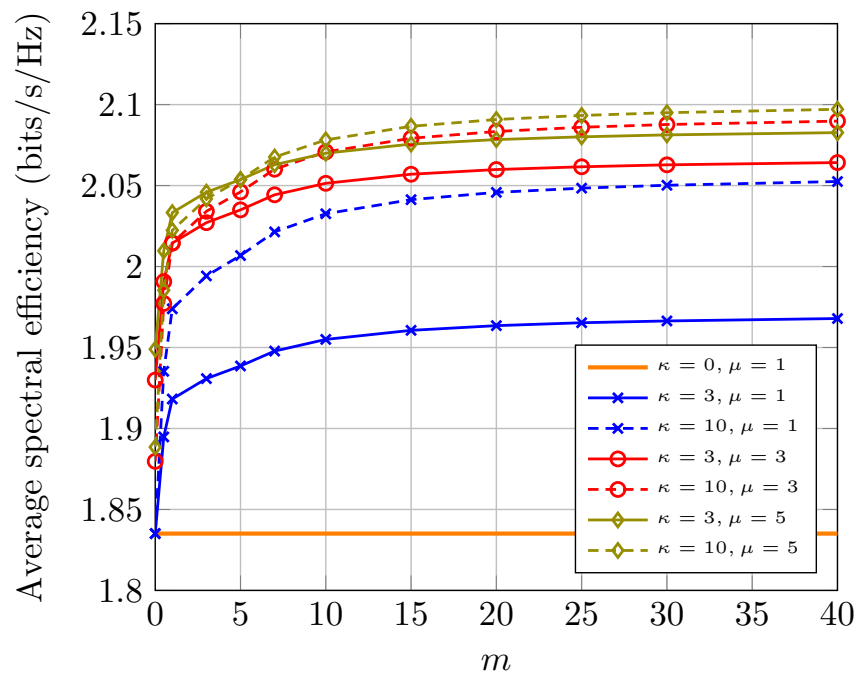

Figure 7: Impact of $m$ on the spatially averaged spectral efficiency $(\eta=3.8)$.

is higher as $m$ decreases. The remainder parameters are set to: LoS environments $(\kappa=3,10)$ with number of clusters $\mu=1$, 3,5 , and NLoS scenario $(\kappa=0, \mu=1)$. It is straightforward to see that increasing $m$ implies increasing $\bar{C}$, because the LoS fluctuation diminishes. The effect of $m$ is more noteworthy for higher values of $\kappa$, as shown in Fig. 7. This makes sense as for a larger $\kappa$, the amount of power arriving from the dominant specular components is more relevant, and hence any fluctuation has a relatively larger impact. For $m<5$, the impact is considerable, but for greater $m$, changes are slight, especially above 20 where they can be considered almost negligible. We also note that NLoS situations are clearly not affected by $m$, as expected.

\section{Impact of $\kappa$}

Here the influence of increasing the power of the main components is studied, from a NLoS environment $(\kappa=0)$ towards higher LoS ones. The remainder parameters are set 


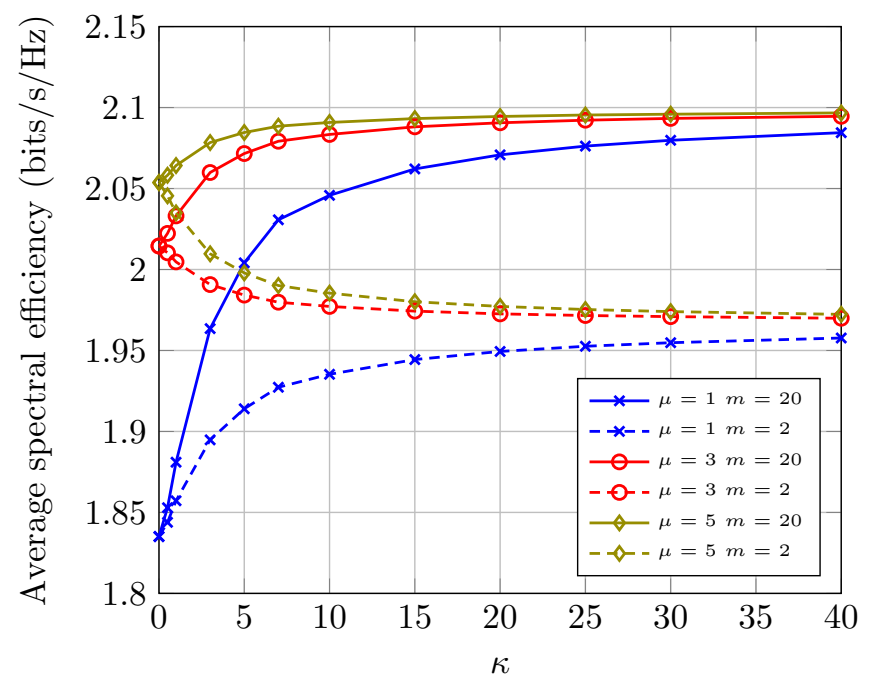

Figure 8: Impact of $\kappa$ on the spatially averaged spectral efficiency $(\eta=3.8)$.

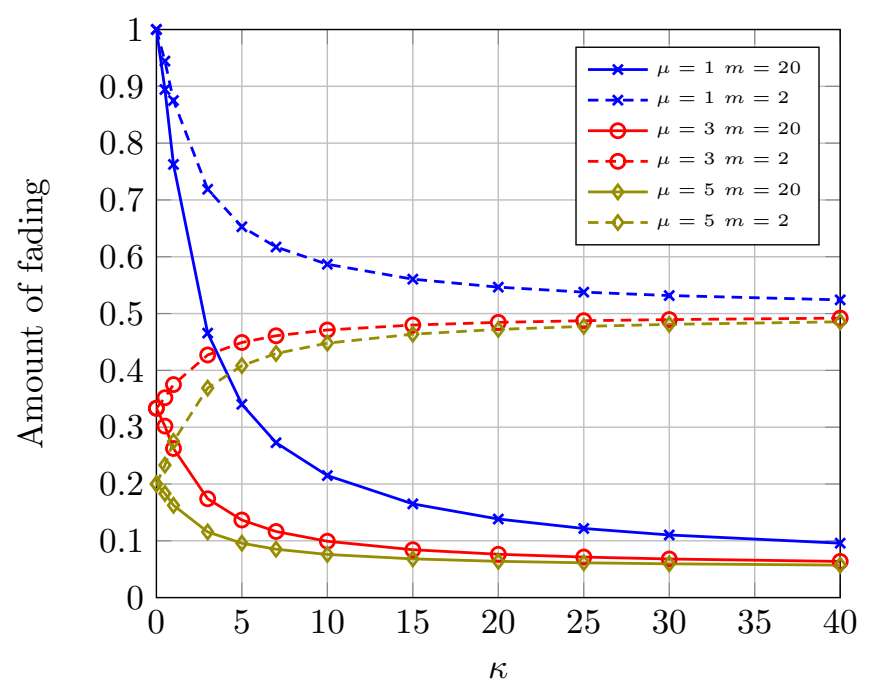

Figure 9: AoF for the same environments than in Fig. 8.

to: fluctuation LoS degree (high, $m=2$; none, $m=20$ ) and number of clusters $(\mu=1,3,5)$. For scenarios with a single cluster, as those in Table I, the higher the $\kappa$, the better the $\bar{C}$, as shown in Fig. 8. But for multicluster situations $(\mu>1)$, when $m$ is high enough, $\kappa$ has a positive impact, but for low $m$, it is just the opposite, and $\bar{C}$ decreases with $\kappa$. The change of tendency happens when $\mu=m$, as observed in [7]. In the AoF expression in (9), this corresponds to $A o F=1 / m$, where the model equals to Nakagami- $m$ fading. The minimum value of $\kappa$ that approaches the maximum $\bar{C}$ decreases with the number of clusters.

To further understanding how AoF is related to the behavior of $\bar{C}$, Fig. 9 presents the corresponding AoFs for the different propagation scenarios considered in Fig. 8. As the AoF is a measure of fading severity, evolution of the AoF and the capacity are inversely proportional.

\section{CONCLUSION}

In this paper, the impact of LoS signals on the spatially average spectral efficiency has been analyzed using the $\kappa-\mu$ shadowed fading model. The analysis has relied on the fact that the large-scale and small-scale fading variations can be split to simplify the mathematical treatment of the problem. With this approach, we have investigated the effect of LoS in the spectral efficiency, through several scenarios that range from heavily fluctuating LoS to deterministic LoS. Additionally, this analysis has shed light on the interplays between fading severity and spectral efficiency through the AoF. Finally, we have studied the effect of the main parameters of the fading distribution on the spectral efficiency and AoF. Future extensions of this work include the analytical derivation of the spectral efficiency expressions here simulated, together with the consideration of different state-of-the-art fading models.

\section{ACKNOWLEDGMENT}

This work has been funded by the Spanish Government and the European Fund for Regional Development FEDER (TEC2016-80090-C2-1-R, TEC2017-87913-R), and Universidad de Málaga.

\section{REFERENCES}

[1] J. An, K. Yang, J. Wu, N. Ye, S. Guo, Z. Liao, "Achieving sustainable ultra-dense heterogeneous network for 5G", IEEE Commun. Mag., vol. 55, no. 12, pp. 84-90, Dec. 2017.

[2] J.G. Andrews, F. Baccelli, R.K. Ganti, "A tractable approach to coverage and rate in cellular networks", IEEE Trans. Commun., vol. 59, no. 11, pp. 3122-3134, Nov. 2011.

[3] H. ElSawy, A. Sultan-Salem, M-S. Alouini, M.Z. Win, "Modeling and analysis of cellular networks using stochastic geometry: A tutorial", IEEE Commun. Surveys \& Tutorials, vol. 19, no. 1, pp. 167-203, 2017.

[4] G. George, R.K. Mungara, A. Lozano, M. Haenggi, "Ergodic Spectral Efficiency in MIMO Cellular Networks", IEEE Trans. on Wireless Communications, vol. 16, no. 5, pp. 2835-2849, Sept. 2017.

[5] José F. Paris, "Statistical Characterization of $\kappa-\mu$ Shadowed Fading Channels", IEEE Trans. on Vehicular Technology,vol.63, no. 2, pp. 518526, Feb. 2014.

[6] Yacoub, M.D., "The $\kappa-\mu$ distribution and the $\eta-\mu$ distribution", IEEE Antennas Propag. Mag.,vol. 49, no. 1, pp. 68-81, 2007.

[7] L. Moreno-Pozas, F. J. Lopez-Martinez, J. F. Paris and E. MartosNaya, "The $\kappa-\mu$ Shadowed Fading Model: Unifying the $\kappa-\mu$ and $\eta-\mu$ Distributions", IEEE Transactions on Vehicular Technology, vol. 65, no. 12, pp. 9630-9641, Dec. 2016.

[8] S. Parthasarathy and R. K. Ganti, "Coverage Analysis in Downlink Poisson Cellular Network With $\kappa-\mu$ Shadowed Fading," IEEE Wireless Communications Letters, vol. 6, no. 1, pp. 10-13, Feb. 2017.

[9] Y. J. Chun, S. L. Cotton, H. S. Dhillon, A. Ghrayeb and M. O. Hasna, "A Stochastic Geometric Analysis of Device-to-Device Communications Operating Over Generalized Fading Channels", IEEE Trans. on Wireless Communications, vol. 16, no. 7, pp. 4151-4165, July 2017.

[10] Y. J. Chun, S. L. Cotton, H. S. Dhillon, F. J. Lopez-Martinez, J. F. Paris and S. K. Yoo, "A Comprehensive Analysis of 5G Heterogeneous Cellular Systems Operating Over $\kappa-\mu$ Shadowed Fading Channels," IEEE Trans. on Wireless Communications, vol. 16, no. 11, pp. 6995-7010, Nov. 2017.

[11] Goldsmith, Andrea. Wireless communications. Cambridge university press, 2005.

[12] F.J Lopez-Martinez, J.F. Paris, J.M. Romero-Jerez "The $\kappa-\mu$ Shadowed Fading Model With Integer Fading Parameters", IEEE Trans. on Vehicular Technology, vol. 66, no. 9, pp. 7653-7662, Sept. 2017.

[13] M.K. Simon and M-S. Alouini, Digital Communication over Fading Channels, John Wiley \& Sons, 2005 